\section{TATRA \\ MOUNTaiNS \\ Mathematical Publications}

DOI: $10.2478 /$ tmmp-2020-0029

Tatra Mt. Math. Publ. 77 (2020), 27-38

\title{
ON THE COMBINATORIAL PROPERTIES OF BIHYPERBOLIC BALANCING NUMBERS
}

\author{
Dorota Bród - Anetta Szynal-Liana — Iwona WŁoch
}

Department of Discrete Mathematics, Rzeszow University of Technology, Rzeszow, POLAND

\begin{abstract}
In this paper, we introduce bihyperbolic balancing and Lucas-balancing numbers. We give some of their properties, among others the Binet formula, Catalan, Cassini, d'Ocagne identities and the generating function.
\end{abstract}

\section{Introduction}

A hyperbolic number is defined as $z=x+\mathbf{h} y$, where $x, y \in \mathbb{R}$ and $\mathbf{h}$ is a unipotent (hyperbolic) imaginary unit such that $\mathbf{h}^{2}=1$ and $\mathbf{h} \neq \pm 1$. Hence the set of hyperbolic numbers is defined as

$$
\mathbb{H}=\left\{z: z=x+\mathbf{h} y, x, y \in \mathbb{R}, \mathbf{h}^{2}=1\right\} .
$$

Hyperbolic imaginary units were introduced in 1848 by James Cockle (see [4] -7]). Let $\mathbb{H}_{2}$ be the set of bihyperbolic numbers defined by

$$
\zeta=x_{0}+j_{1} x_{1}+j_{2} x_{2}+j_{3} x_{3},
$$

where $x_{0}, x_{1}, x_{2}, x_{3} \in \mathbb{R}$ and $j_{1}, j_{2}, j_{3} \notin \mathbb{R}$ are operators such that

and

$$
j_{1}^{2}=j_{2}^{2}=j_{3}^{2}=1
$$

$$
j_{1} j_{2}=j_{2} j_{1}=j_{3}, \quad j_{1} j_{3}=j_{3} j_{1}=j_{2}, \quad j_{2} j_{3}=j_{3} j_{2}=j_{1} .
$$

The addition and multiplication on $\mathbb{H}_{2}$ are commutative and associative. Also, the multiplication is distributive over addition. Hence $\left(\mathbb{H}_{2},+, \cdot\right)$ is a commutative ring. Hyperbolic numbers and bihyperbolic numbers are well-studied in the literature, see [1,11,12]. In this paper we introduce bihyperbolic balancing numbers and bihyperbolic Lucas-balancing numbers.

(C) 2020 Mathematical Institute, Slovak Academy of Sciences. 2010 Mathematics Subject Classification: 11B37, 11 B39.

Keywords: balancing numbers, Lucas-balancing numbers, Binet formula, hyperbolic numbers, bihyperbolic numbers, recurrence relations.

Licensed under the Creative Commons Attribution-NC-ND4.0 International Public License. 
The sequence of balancing numbers, denoted by $\left\{B_{n}\right\}$, was introduced by Behera and Panda in 2. A balancing number $n$ with balancer $r$ is the solution of the Diophantine equation

$$
1+2+\cdots+(n-1)=(n+1)+(n+2)+\cdots+(n+r) .
$$

For example, 6 is a balancing number with balancer 2, 35 is a balancing number with balancer 14. In [2] it was proved that the balancing numbers satisfy the following recurrence relation

$$
B_{n}=6 B_{n-1}-B_{n-2} \text { for } n \geq 2
$$

with initial conditions $B_{0}=0, B_{1}=1$. The sequence of balancing numbers is given by Binet formula

$$
B_{n}=\frac{r_{1}^{n}-r_{2}^{n}}{r_{1}-r_{2}}
$$

where $r_{1}, r_{2}$ are the roots of the characteristic equation $r^{2}-6 r+1=0$, associated with the recurrence relation (3), i.e.,

$$
r_{1}=3+2 \sqrt{2}, \quad r_{2}=3-2 \sqrt{2} .
$$

Note that

$$
\begin{aligned}
r_{1}+r_{2} & =6, \\
r_{1}-r_{2} & =4 \sqrt{2}, \\
r_{1} r_{2} & =1 .
\end{aligned}
$$

It is well known that $n$ is a balancing number if and only if $n^{2}$ is a triangular number, i.e., $8 n^{2}+1$ is a perfect square, see [2]. In [8], the author introduced Lucas-balancing numbers, defined as follows: if $B_{n}$ is a balancing number, the number $C_{n}=\sqrt{8 B_{n}^{2}+1}$ is called a Lucas-balancing number. The sequence $\left\{C_{n}\right\}$ of Lucas-balancing numbers is also defined by the recurrence relation

$$
C_{n}=6 C_{n-1}-C_{n-2} \text { for } n \geq 2
$$

with initial terms $C_{0}=1, C_{1}=3$. The Binet formula for the Lucas-balancing numbers has the following form

$$
C_{n}=\frac{1}{2}\left(r_{1}^{n}+r_{2}^{n}\right)
$$

where $r_{1}, r_{2}$ are given by (5). 
TABLE 1.

\begin{tabular}{c||cccccccc}
$n$ & 0 & 1 & 2 & 3 & 4 & 5 & 6 & 7 \\
\hline \hline$B_{n}$ & 0 & 1 & 6 & 35 & 204 & 1189 & 6930 & 40391 \\
$C_{n}$ & 1 & 3 & 17 & 99 & 577 & 3363 & 19601 & 114243
\end{tabular}

The Table 1 includes initial terms of the balancing and Lucas-balancing numbers for $n=0,1, \ldots, 7$. Many interesting properties of the balancing and Lucasbalancing numbers are presented in $[2,3,8,10,13]$. We give some of them.

$$
\begin{aligned}
B_{n-r} B_{n+r}-B_{n}^{2} & =-B_{r}^{2} & & \text { (Catalan identity), } \\
C_{n-r} C_{n+r}-C_{n}^{2} & =C_{r}^{2}-1 & & \text { (Catalan identity), } \\
B_{n-1} B_{n+1}-B_{n}^{2} & =-1 & & \text { (Cassini identity), } \\
C_{n-1} C_{n+1}-C_{n}^{2} & =8 & & \text { (Cassini identity) }, \\
B_{m} B_{n+1}-B_{m+1} B_{n} & =B_{m-n} & & \text { (d'Ocagne identity) }, \\
C_{m} C_{n+1}-C_{m+1} C_{n} & =-8 B_{m-n} & & \text { (d'Ocagne identity). }
\end{aligned}
$$

In this paper we use the following identities:

$$
\begin{gathered}
\sum_{i=0}^{n} B_{i}=\frac{B_{n+1}-B_{n}-1}{4} \\
\sum_{i=0}^{n} C_{i}=\frac{C_{n+1}-C_{n}+2}{4} \\
3 B_{n}-B_{n-1}=C_{n} \\
B_{n+2}-B_{n-2}=12 C_{n} .
\end{gathered}
$$

\section{Bihyperbolic balancing numbers}

In this section, we introduce bihyperbolic balancing numbers and bihyperbolic Lucas-balancing numbers.

Let $n \geq 0$ be an integer. We define the $n$th bihyperbolic balancing number $B h B_{n}$ as

$$
B h B_{n}=B_{n}+j_{1} B_{n+1}+j_{2} B_{n+2}+j_{3} B_{n+3},
$$

where $B_{n}$ is $n$th balancing number. 
In the same way, we define the bihyperbolic Lucas-balancing numbers $B h C_{n}$

$$
B h C_{n}=C_{n}+j_{1} C_{n+1}+j_{2} C_{n+2}+j_{3} C_{n+3},
$$

where $C_{n}$ is $n$th Lucas-balancing number.

Using (15), (16) and Table 1, we get

$$
\begin{aligned}
B h B_{0} & =j_{1}+6 j_{2}+35 j_{3}, \\
B h B_{1} & =1+6 j_{1}+35 j_{2}+204 j_{3}, \\
B h B_{2} & =6+35 j_{1}+204 j_{2}+1189 j_{3}, \\
B h B_{3} & =35+204 j_{1}+1189 j_{2}+6930 j_{3} \\
& \vdots \\
B h C_{0} & =1+3 j_{1}+17 j_{2}+99 j_{3}, \\
B h C_{1} & =3+17 j_{1}+99 j_{2}+577 j_{3}, \\
B h C_{2} & =17+99 j_{1}+577 j_{2}+3363 j_{3}, \\
B h C_{3} & =99+577 j_{1}+3363 j_{2}+19601 j_{3}
\end{aligned}
$$

By the definition of bihyperbolic balancing and Lucas-balancing numbers, we get the following recurrence relations.

Theorem 2.1. Let $n \geq 2$ be an integer. Then

(i) $B h B_{n}=6 B h B_{n-1}-B h B_{n-2}$,

(ii) $B h C_{n}=6 B h C_{n-1}-B h C_{n-2}$,

where $B h B_{0}, B h B_{1}, B h C_{0}, B h C_{1}$ are given by (17), (18), respectively.

P r o of. (i) Using (15) and (3), we have

$$
\begin{aligned}
6 B h B_{n-1}-B h B_{n-2}= & 6\left(B_{n-1}+j_{1} B_{n}+j_{2} B_{n+1}+j_{3} B_{n+2}\right) \\
& -\left(B_{n-2}+j_{1} B_{n-1}+j_{2} B_{n}+j_{3} B_{n+1}\right) \\
= & 6 B_{n-1}-B_{n-2}+j_{1}\left(6 B_{n}-B_{n-1}\right) \\
& +j_{2}\left(6 B_{n+1}-B_{n}\right)+j_{3}\left(6 B_{n+2}-B_{n+1}\right) \\
= & B_{n}+j_{1} B_{n+1}+j_{2} B_{n+2}+j_{3} B_{n+3}=B h B_{n} .
\end{aligned}
$$

We omit the proof of (ii). 
TheOREM 2.2. Let $n \geq 1$ be an integer. Then

$$
B h C_{n}=3 B h B_{n}-B h B_{n-1} .
$$

P r o o f. By formulas (15) and (13), we have

$$
\begin{aligned}
3 B h B_{n}-B h B_{n-1}= & 3\left(B_{n}+j_{1} B_{n+1}+j_{2} B_{n+2}+j_{3} B_{n+3}\right) \\
& -B_{n-1}-j_{1} B_{n}-j_{2} B_{n+1}-j_{3} B_{n+2} \\
= & 3 B_{n}-B_{n-1}+j_{1}\left(3 B_{n+1}-B_{n}\right) \\
& +j_{2}\left(3 B_{n+2}-B_{n+1}\right) \\
& +j_{3}\left(3 B_{n+3}-B_{n+2}\right) \\
= & C_{n}+j_{1} C_{n+1}+j_{2} C_{n+2}+j_{3} C_{n+3} \\
= & B h C_{n} .
\end{aligned}
$$

TheOrem 2.3. Let $n \geq 2$ be an integer. Then

$$
B h B_{n+2}-B h B_{n-2}=12 B h C_{n} .
$$

P r o o f. Using (15), (14) and (16), we obtain

$$
\begin{aligned}
B h B_{n+2}-B h B_{n-2}= & B_{n+2}+j_{1} B_{n+3}+j_{2} B_{n+4}+j_{3} B_{n+5} \\
& -B_{n-2}-j_{1} B_{n-1}-j_{2} B_{n}-j_{3} B_{n+1} \\
= & B_{n+2}-B_{n-2}+j_{1}\left(B_{n+3}-B_{n-1}\right) \\
& +j_{2}\left(B_{n+4}-B_{n}\right)+j_{3}\left(B_{n+5}-B_{n+1}\right) \\
= & 12\left(C_{n}+j_{1} C_{n+1}+j_{2} C_{n+2}+j_{3} C_{n+3}\right) \\
= & 12 B h C_{n} .
\end{aligned}
$$

Theorem 2.4. Let $n \geq 0, r \geq 1$ be integers. Then

$$
\begin{aligned}
& B h B_{n}-j_{1} B h B_{n+1}-j_{2} B h B_{n+2}-j_{3} B h B_{n+3}= \\
& B_{n}-B_{n+2}-B_{n+4}+B_{n+6}-2 j_{3} B h B_{n+3} .
\end{aligned}
$$


P r o of. Using multiplication rules (11) and (2), we obtain

$$
\begin{aligned}
B h & B_{n}-j_{1} B h B_{n+1}-j_{2} B h B_{n+2}-j_{3} B h B_{n+3} \\
= & B_{n}+j_{1} B_{n+1}+j_{2} B_{n+2}+j_{3} B_{n+3} \\
& -j_{1}\left(B_{n+1}+j_{1} B_{n+2}+j_{2} B_{n+3}+j_{3} B_{n+4}\right) \\
& -j_{2}\left(B_{n+2}+j_{1} B_{n+3}+j_{2} B_{n+4}+j_{3} B_{n+5}\right) \\
& -j_{3}\left(B_{n+3}+j_{1} B_{n+4}+j_{2} B_{n+5}+j_{3} B_{n+6}\right) \\
= & B_{n}+j_{1} B_{n+1}+j_{2} B_{n+2}+j_{3} B_{n+3} \\
& -j_{1} B_{n+1}-B_{n+2}-j_{3} B_{n+3}-j_{2} B_{n+4} \\
& -j_{2} B_{n+2}-j_{3} B_{n+3}-B_{n+4}-j_{1} B_{n+5} \\
& -j_{3} B_{n+3}-j_{2} B_{n+4}-j_{1} B_{n+5}-B_{n+6} \\
= & B_{n}-B_{n+2}-B_{n+4}-B_{n+6} \\
& -2\left(j_{1} B_{n+5}+j_{2} B_{n+4}+j_{3} B_{n+3}\right) \\
= & B_{n}-B_{n+2}-B_{n+4}+B_{n+6} \\
& -2 j_{3}\left(B_{n+3}+j_{1} B_{n+4}+j_{2} B_{n+5}+j_{3} B_{n+6}\right) \\
= & B_{n}-B_{n+2}-B_{n+4}+B_{n+6}-2 j_{3} B h B_{n+3},
\end{aligned}
$$

which ends the proof.

The next theorem gives the Binet formulas for the bihyperbolic balancing and Lucas-balancing numbers.

Theorem 2.5. Let $n \geq 0$ be an integer. Then

$$
\begin{aligned}
B h B_{n} & =\frac{\hat{r_{1}} r_{1}^{n}-\hat{r_{2}} r_{2}^{n}}{r_{1}-r_{2}}, \\
B h C_{n} & =\frac{\hat{r_{1}} r_{1}^{n}+\hat{r_{2}} r_{2}^{n}}{2},
\end{aligned}
$$

where $r_{1}$ and $r_{2}$ are given by (5) and

$$
\begin{aligned}
& \hat{r_{1}}=1+j_{1} r_{1}+j_{2} r_{1}^{2}+j_{3} r_{1}^{3}, \\
& \hat{r_{2}}=1+j_{1} r_{2}+j_{2} r_{2}^{2}+j_{3} r_{2}^{3} .
\end{aligned}
$$


P r o of. By formula (4), we get

$$
\begin{aligned}
B h B_{n}= & B_{n}+j_{1} B_{n+1}+j_{2} B_{n+2}+j_{3} B_{n+3} \\
= & \frac{1}{r_{1}-r_{2}}\left[r_{1}^{n}-r_{2}^{n}+j_{1}\left(r_{1}^{n+1}-r_{2}^{n+1}\right)\right. \\
& \left.+j_{2}\left(r_{1}^{n+2}-r_{2}^{n+2}\right)+j_{3}\left(r_{1}^{n+3}-r_{2}^{n+3}\right)\right] \\
= & \frac{1}{r_{1}-r_{2}}\left[r_{1}^{n}\left(1+j_{1} r_{1}+j_{2} r_{1}^{2}+j_{3} r_{1}^{3}\right)\right. \\
& \left.-r_{2}^{n}\left(1+j_{1} r_{2}+j_{2} r_{2}^{2}+j_{3} r_{2}^{3}\right)\right] \\
= & \frac{\hat{r_{1}} r_{1}^{n}-\hat{r_{2}} r_{2}^{n}}{r_{1}-r_{2}} .
\end{aligned}
$$

We omit the proof of (20).

By simple calculations, we obtain

$$
\begin{aligned}
\hat{r_{1}} \hat{r_{2}}=\hat{r_{2}} \hat{r_{1}}= & 1+r_{1} r_{2}+\left(r_{1} r_{2}\right)^{2} \\
& +\left(r_{1} r_{2}\right)^{3}+j_{1}\left(r_{1}+r_{2}\right)\left(1+\left(r_{1} r_{2}\right)^{2}\right) \\
& +j_{2}\left(r_{1}^{2}+r_{2}^{2}\right)\left(1+r_{1} r_{2}\right) \\
& +j_{3}\left(r_{1}^{3}+r_{2}^{3}+r_{1} r_{2}\left(r_{1}+r_{2}\right)\right) .
\end{aligned}
$$

Using formulas (6) - (8) we get

$$
\begin{aligned}
& r_{1}^{2}+r_{2}^{2}=\left(r_{1}+r_{2}\right)^{2}-2 r_{1} r_{2}=34 \\
& r_{1}^{3}+r_{2}^{3}=\left(r_{1}+r_{2}\right)^{3}-3 r_{1} r_{2}\left(r_{1}+r_{2}\right)=198 .
\end{aligned}
$$

Thus

$$
\begin{aligned}
\hat{r_{1}} \hat{r_{2}}=\hat{r_{2}} \hat{r_{1}} & =4+12 j_{1}+68 j_{2}+204 j_{3} \\
& =4 B h C_{0}-192 j_{3} .
\end{aligned}
$$

\section{Some identities for the bihyperbolic balancing and Lucas-balancing numbers}

In this section, we give some identities such as Catalan, Cassini and d'Ocagne identities for the bihyperbolic balancing and Lucas-balancing numbers. These identities are easily proved using the Binet formulas (19) and (20).

Theorem 3.1 (Catalan identities). Let $n \geq 0, r \geq 0$ be integers such that $n \geq r$. Then

(i) $B h B_{n-r} B h B_{n+r}-\left(B h B_{n}\right)^{2}=-\hat{r_{1}} \hat{r_{2}}\left(\frac{r_{1}^{r}-r_{2}^{r}}{r_{1}-r_{2}}\right)^{2}$,

(ii) $B h C_{n-r} B h C_{n+r}-\left(B h C_{n}\right)^{2}=\frac{1}{4} \hat{r_{1}} \hat{r_{2}}\left(r_{2}^{r}-r_{1}^{r}\right)^{2}$. 
Proof.

(i) By formula (19), we get

$$
\begin{aligned}
& B h B_{n-r} B h B_{n+r}-\left(B h B_{n}\right)^{2} \\
& =\frac{\left(\hat{r_{1}} r_{1}^{n-r}-\hat{r_{2}} r_{2}^{n-r}\right)\left(\hat{r_{1}} r_{1}^{n+r}-\hat{r_{2}} r_{2}^{n+r}\right)-\left(\hat{r_{1}} r_{1}^{n}-\hat{r_{2}} r_{2}^{n}\right)^{2}}{\left(r_{1}-r_{2}\right)^{2}} \\
& =\frac{1}{\left(r_{1}-r_{2}\right)^{2}}\left[\hat{r_{1}} \hat{r_{2}}\left(r_{1}^{n} r_{2}^{n}\right)\left(1-\left(\frac{r_{2}}{r_{1}}\right)^{r}\right)+\hat{r_{2}} \hat{r_{1}}\left(r_{1}^{n} r_{2}^{n}\right)\left(1-\left(\frac{r_{1}}{r_{2}}\right)^{r}\right)\right] .
\end{aligned}
$$

Using the fact that $r_{1} r_{2}=1$, we have

$$
\begin{aligned}
& B h B_{n-r} B h B_{n+r}-\left(B h B_{n}\right)^{2} \\
& =\frac{1}{\left(r_{1}-r_{2}\right)^{2}}\left(\hat{r_{1}} \hat{r_{2}} \frac{r_{1}^{r}-r_{2}^{r}}{r_{1}^{r}}+\hat{r_{2}} \hat{r_{1}} \frac{r_{2}^{r}-r_{1}^{r}}{r_{2}^{r}}\right)=\frac{\hat{r_{1}} \hat{r_{2}}\left(r_{1}^{r}-r_{2}^{r}\right) r_{2}^{r}-\hat{r_{2}} \hat{r_{1}}\left(r_{1}^{r}-r_{2}^{r}\right) r_{1}^{r}}{\left(r_{1}-r_{2}\right)^{2}} \\
& =\frac{\left(r_{1}^{r}-r_{2}^{r}\right)\left(\hat{r_{1}} \hat{r_{2}} r_{2}^{r}-\hat{r_{2}} \hat{r_{1}} r_{1}^{r}\right)}{\left(r_{1}-r_{2}\right)^{2}}=-\hat{r_{1}} \hat{r_{2}}\left(\frac{r_{1}^{r}-r_{2}^{r}}{r_{1}-r_{2}}\right)^{2} .
\end{aligned}
$$

(ii) In the same way, using formula (20), one can easily prove the result.

By Theorem 3.1, for $r=1$ we get the Cassini identities for the bihyperbolic balancing and Lucas-balancing numbers.

Corollary 3.2. For $n \geq 1$

(i) $B h B_{n-1} B h B_{n+1}-\left(B h B_{n}\right)^{2}=-\hat{r_{1}} \hat{r_{2}}$,

(ii) $B h C_{n-1} B h C_{n+1}-\left(B h C_{n}\right)^{2}=8 \hat{r_{1}} \hat{r_{2}}$.

Theorem 3.3 (d'Ocagne identities). Let $m \geq 0, n \geq 0$ be integers such that $m \geq n$. Then

(i) $B h B_{m} B h B_{n+1}-B h B_{m+1} B h B_{n}=\frac{\hat{r_{1}} \hat{r_{2}}\left(r_{1}^{m-n}-r_{2}^{m-n}\right)}{r_{1}-r_{2}}$,

(ii) $B h C_{m} B h C_{n+1}-B h C_{m+1} B h C_{n}=\frac{1}{4} \hat{r_{1}} \hat{r_{2}}\left(r_{1}^{m-n}-r_{2}^{m-n}\right)\left(r_{2}-r_{1}\right)$.

Proof.

(i) By formulas (19) and (44), we get

$$
\begin{aligned}
& B h B_{m} B h B_{n+1}-B h B_{m+1} B h B_{n} \\
& =\frac{\left(\hat{r_{1}} r_{1}^{m}-\hat{r_{2}} r_{2}^{m}\right)\left(\hat{r_{1}} r_{1}^{n+1}-\hat{r_{2}} r_{2}^{n+1}\right)-\left(\hat{r_{1}} r_{1}^{m+1}-\hat{r_{2}} r_{2}^{m+1}\right)\left(\hat{r_{1}} r_{1}^{n}-\hat{r_{2}} r_{2}^{n}\right)}{\left(r_{1}-r_{2}\right)^{2}} \\
& =\frac{1}{\left(r_{1}-r_{2}\right)^{2}}\left[\hat{r_{1}} \hat{r_{2}}\left(r_{1}^{m+1} r_{2}^{n}-r_{1}^{m} r_{2}^{n+1}\right)+\hat{r_{2}} \hat{r_{1}}\left(r_{1}^{n} r_{2}^{m+1}-r_{1}^{n+1} r_{2}^{m}\right)\right] \\
& =\frac{1}{\left(r_{1}-r_{2}\right)^{2}}\left(r_{1} r_{2}\right)^{n}\left[\hat{r_{1}} \hat{r_{2}}\left(r_{1}-r_{2}\right) r_{1}^{m-n}+\hat{r_{2}} \hat{r_{1}}\left(r_{2}-r_{1}\right) r_{2}^{m-n}\right] \\
& =\frac{\hat{r_{1}} \hat{r_{2}} r_{1}^{m-n}-\hat{r_{2}} \hat{r_{1}} r_{2}^{m-n}}{r_{1}-r_{2}}=\frac{\hat{r_{1}} \hat{r_{2}}\left(r_{1}^{m-n}-r_{2}^{m-n}\right)}{r_{1}-r_{2}} \text {. }
\end{aligned}
$$

(ii) By formula (201), we have

$$
\begin{aligned}
& B h C_{m} B h C_{n+1}-B h C_{m+1} B h C_{n} \\
& =\frac{1}{4}\left(r_{1} r_{2}\right)^{n}\left(\hat{r_{1}} \hat{r_{2}}\left(r_{2}-r_{1}\right) r_{1}^{m-n}+\hat{r_{2}} \hat{r_{1}}\left(r_{1}-r_{2}\right) r_{2}^{m-n}\right) \\
& =\frac{1}{4} \hat{r_{1}} \hat{r_{2}}\left(r_{1}^{m-n}-r_{2}^{m-n}\right)\left(r_{2}-r_{1}\right) .
\end{aligned}
$$


Theorem 3.4. Let $m \geq 0, n \geq 0$ be integers. Then

(i) $B h B_{m} B h C_{n}-B h C_{m} B h B_{n}=\frac{\hat{r_{1}} \hat{r_{2}}\left(r_{1}^{m-n}-r_{2}^{m-n}\right)}{r_{1}-r_{2}}$,

(ii) $B h B_{m} B h C_{n}+B h C_{m} B h B_{n}=\frac{\left(\hat{r_{1}}\right)^{2} r_{1}^{m+n}-\left(\hat{r_{2}}\right)^{2} r_{2}^{m+n}}{r_{1}-r_{2}}$.

Proof.

(i)

$$
\begin{aligned}
& B h B_{m} B h C_{n}-B h C_{m} B h B_{n} \\
& =\frac{1}{2\left(r_{1}-r_{2}\right)}\left[\left(\hat{r_{1}} r_{1}^{m}-\hat{r_{2}} r_{2}^{m}\right)\left(\hat{r_{1}} r_{1}^{n}+\hat{r_{2}} r_{2}^{n}\right)-\left(\hat{r_{1}} r_{1}^{m}+\hat{r_{2}} r_{2}^{m}\right)\left(\hat{r_{1}} r_{1}^{n}-\hat{r_{2}} r_{2}^{n}\right)\right] \\
& =\frac{1}{2\left(r_{1}-r_{2}\right)}\left[2 \hat{r_{1}} \hat{r_{2}} r_{1}^{m} r_{2}^{n}-2 \hat{r_{2}} r_{1}^{n} r_{2}^{m}\right] \\
& =\frac{1}{r_{1}-r_{2}}\left[\left(r_{1} r_{2}\right)^{n}\left(\hat{r_{1}} \hat{r_{2}} r_{1}^{m-n}-\hat{r_{2}} \hat{r_{1}} r_{2}^{m-n}\right)\right] \\
& =\frac{\hat{r_{1}} \hat{r_{2}}\left(r_{1}^{m-n}-r_{2}^{m-n}\right)}{r_{1}-r_{2}} .
\end{aligned}
$$

(ii)

$$
\begin{aligned}
& B h B_{m} B h C_{n}+B h C_{m} B h B_{n} \\
& =\frac{1}{2\left(r_{1}-r_{2}\right)}\left[\left(\hat{r_{1}} r_{1}^{m}-\hat{r_{2}} r_{2}^{m}\right)\left(\hat{r_{1}} r_{1}^{n}+\hat{r_{2}} r_{2}^{n}\right)+\left(\hat{r_{1}} r_{1}^{m}+\hat{r_{2}} r_{2}^{m}\right)\left(\hat{r_{1}} r_{1}^{n}-\hat{r_{2}} r_{2}^{n}\right)\right] \\
& =\frac{1}{2\left(r_{1}-r_{2}\right)}\left[2\left(\hat{r_{1}}\right)^{2} r_{1}^{m+n}-2\left(\hat{r_{2}}\right)^{2} r_{2}^{m+n}\right] \\
& =\frac{\left(\hat{r_{1}}\right)^{2} r_{1}^{m+n}-\left(\hat{r_{2}}\right)^{2} r_{2}^{m+n}}{r_{1}-r_{2}} .
\end{aligned}
$$

Theorem 3.5. Let $n \geq 0, r \geq 0, s \geq 0$ be integers. Then

$$
B h B_{n+r} B h C_{n+s}-B h B_{n+s} B h C_{n+r}=\frac{\hat{r_{1}} \hat{r_{2}}\left(r_{1}^{r} r_{2}^{s}-r_{1}^{s} r_{2}^{r}\right)}{r_{1}-r_{2}} .
$$

P r o o f. Using formulas (19) and (20), we have

$$
\begin{aligned}
& B h B_{n+r} B h C_{n+s}-B h B_{n+s} B h C_{n+r} \\
&= \frac{1}{2\left(r_{1}-r_{2}\right)}\left[\left(\hat{r_{1}} r_{1}^{n+r}-\hat{r_{2}} r_{2}^{n+r}\right)\left(\hat{r_{1}} r_{1}^{n+s}+\hat{r_{2}} r_{2}^{n+s}\right)\right. \\
&\left.-\left(\hat{r_{1}} r_{1}^{n+s}-\hat{r_{2}} r_{2}^{n+s}\right)\left(\hat{r_{1}} r_{1}^{n+r}+\hat{r_{2}} r_{2}^{n+r}\right)\right] \\
&= \frac{1}{2\left(r_{1}-r_{2}\right)}\left[\hat{r_{1}} \hat{r_{2}} r_{1}^{n+r} r_{2}^{n+s}-\hat{r_{1}} \hat{r_{2}} r_{1}^{n+s} r_{2}^{n+r}\right. \\
&\left.+\hat{r_{2}} \hat{r_{1}} r_{1}^{n+r} r_{2}^{n+s}-\hat{r_{2}} \hat{r_{1}} r_{1}^{n+s} r_{2}^{n+r}\right] \\
&= \frac{1}{2\left(r_{1}-r_{2}\right)}\left[\left(r_{1} r_{2}\right)^{n}\left(\hat{r_{1}} \hat{r_{2}}+\hat{r_{2}} \hat{r_{1}}\right)\left(r_{1}^{r} r_{2}^{s}-r_{1}^{s} r_{2}^{r}\right)\right] \\
&= \frac{\hat{r_{1}}\left(\hat{r_{1}} r_{2}^{s}-r_{1}^{s} r_{2}^{r}\right)}{r_{1}-r_{2}} .
\end{aligned}
$$


TheOrem 3.6. Let $n \geq 0$ be an integer. Then

$$
\left(B h C_{n}\right)^{2}-8\left(B h B_{n}\right)^{2}=\hat{r_{1}} \hat{r_{2}} .
$$

P r o o f. By (20) and (19) we get

$$
\begin{aligned}
\left(B h C_{n}\right)^{2}-8\left(B h B_{n}\right)^{2} & =\left(\frac{\hat{r_{1}} r_{1}^{n}+\hat{r_{2}} r_{2}^{n}}{2}\right)^{2}-8\left(\frac{\hat{r_{1}}}{4 \sqrt{r_{1}^{n}}-\hat{r_{2}} r_{2}^{n}}\right)^{2} \\
& =\frac{1}{4}\left[\left(r_{1} r_{2}\right)^{n} 2\left(\hat{r_{1}} \hat{r_{2}}+\hat{r_{2}} \hat{r_{1}}\right)\right]=\hat{r_{1}} \hat{r_{2}} .
\end{aligned}
$$

The following theorem gives a summation formula for the bihyperbolic balancing numbers.

TheOREm 3.7. Let $n \geq 0$ be an integer. Then

$$
\sum_{i=0}^{n} B h B_{i}=\frac{1}{4}\left(B h B_{n+1}-B h B_{n}-\left(1+j_{1}+j_{2}+j_{3}\right)\right)-\left(j_{2}+7 j_{3}\right) .
$$

Pr o of. By formula (11), we have

$$
\begin{aligned}
\sum_{i=0}^{n} B h B_{i}= & \sum_{i=0}^{n}\left(B_{i}+j_{1} B_{i+1}+j_{2} B_{i+2}+j_{3} B_{i+3}\right) \\
= & \sum_{i=0}^{n} B_{i}+j_{1} \sum_{i=0}^{n} B_{i+1}+j_{2} \sum_{i=0}^{n} B_{i+2}+j_{3} \sum_{i=0}^{n} B_{i+3} \\
= & \frac{1}{4}\left(B_{n+1}-B_{n}-1\right)+j_{1}\left(\frac{1}{4}\left(B_{n+2}-B_{n+1}-1\right)-B_{0}\right) \\
& +j_{2}\left(\frac{1}{4}\left(B_{n+3}-B_{n+2}-1\right)-B_{0}-B_{1}\right) \\
& +j_{3}\left(\frac{1}{4}\left(B_{n+4}-B_{n+3}-1\right)-B_{0}-B_{1}-B_{2}\right) \\
= & \frac{1}{4}\left(B_{n+1}+j_{1} B_{n+2}+j_{2} B_{n+3}+j_{3} B_{n+4}\right. \\
& \left.-\left(B_{n}+j_{1} B_{n+1}+j_{2} B_{n+2}+j_{3} B_{n+3}\right)-\left(1+j_{1}+j_{2}+j_{3}\right)\right) \\
& -j_{1} B_{0}-j_{2}\left(B_{0}+B_{1}\right)-j_{3}\left(B_{0}+B_{1}+B_{2}\right) .
\end{aligned}
$$

Hence

$$
\sum_{i=0}^{n} B h B_{i}=\frac{1}{4}\left(B h B_{n+1}-B h B_{n}-\left(1+j_{1}+j_{2}+j_{3}\right)\right)-\left(j_{2}+7 j_{3}\right) .
$$

In the same way, using formula (12), we can prove the next theorem.

TheOrem 3.8. Let $n \geq 0$ be an integer. Then

$$
\sum_{i=0}^{n} B h C_{i}=\frac{1}{4}\left(B h C_{n+1}-B h C_{n}+2\left(1+j_{1}+j_{2}+j_{3}\right)\right)-\left(j_{1}+4 j_{2}+21 j_{3}\right) .
$$




\section{Generating functions}

In [2] and [14, the following theorems were proved.

TheOREM 4.1. [2] The generating function of the balancing sequence $\left\{B_{n}\right\}$ has the following form

$$
G\left(B_{n} ; x\right)=\frac{x}{1-6 x+x^{2}} .
$$

TheOREM 4.2. [14] The generating function of the Lucas-balancing sequence $\left\{C_{n}\right\}$ has the following form

$$
G\left(C_{n} ; x\right)=\frac{1-3 x}{1-6 x+x^{2}} .
$$

Now we will give the generating functions for the bihyperbolic balancing and the Lucas-balancing numbers.

THEOREM 4.3. The generating function of the bihyperbolic balancing sequence has the following form

$$
g(x)=\frac{x+j_{1}+(6-x) j_{2}+(35-6 x) j_{3}}{1-6 x+x^{2}} .
$$

P r o of. Let

$$
g(x)=B h B_{0}+B h B_{1} x+B h B_{2} x^{2}+\cdots+B h B_{n} x^{n}+\cdots
$$

be the generating function of the bihyperbolic balancing sequence. Hence we have

$$
\begin{aligned}
& 6 x g(x)=6 B h B_{0} x+6 B h B_{1} x^{2}+6 B h B_{2} x^{3}+\cdots+6 B h B_{n-1} x^{n}+\cdots, \\
& x^{2} g(x)=B h B_{0} x^{2}+B h B_{1} x^{3}+B h B_{2} x^{4}+\cdots+B h B_{n-2} x^{n}+\cdots
\end{aligned}
$$

Using the recurrence $B h B_{n}=6 B h B_{n-1}-B h B_{n-2}$ and fact that the coefficients of $x^{n}$ for $n \geq 2$ are equal to zero, we get

Thus

$$
g(x)-6 x g(x)+x^{2} g(x)=B h B_{0}+\left(B h B_{1}-6 B h B_{0}\right) x .
$$

$$
g(x)=\frac{B h B_{0}+\left(B h B_{1}-6 B h B_{0}\right) x}{1-6 x+x^{2}} .
$$

Using (17), we obtain

$g(x)=\frac{j_{1}+6 j_{2}+35 j_{3}+\left(1-j_{2}-6 j_{3}\right) x}{1-6 x+x^{2}}=\frac{x+j_{1}+(6-x) j_{2}+(35-6 x) j_{3}}{1-6 x+x^{2}}$.

In the same way we can prove the next theorem.

TheOREM 4.4. The generating function of the bihyperbolic Lucas-balancing sequence has the following form

$$
f(x)=\frac{1-3 x+(3-x) j_{1}+(17-3 x) j_{2}+(99-17 x) j_{3}}{1-6 x+x^{2}} .
$$




\title{
REFERENCES
}

[1] BILGIN, M.-ERSOY, S.: Algebraic Properties of Bihyperbolic Numbers, Adv. Appl. Clifford Algebr. 30 (2020), no. 1, 1-13.

[2] BEHERA, A.-PANDA, G. K.: On the square roots of triangular numbers, Fibonacci Quart. 37 (1999), no. 2, 98-105.

[3] CATARINO, P.-CAMPOS, H.-VASCO, P.: On some identities for balancing and cobalancing numbers, Ann. Math. Inform. 45 (2015), 11-24.

[4] COCKLE, J.: On certain functions resembling quaternions, and on a new imaginary in algebra, Lond. Edinb. Dubl. Phil. Mag. 33 (1848), 435-439.

[5] COCKLE, J.: On a new imaginary in algebra, Lond. Edinb. Dubl. Phil. Mag. 34 (1849), $37-47$.

[6] COCKLE, J.: On the symbols of algebra, and on the theory of tesarines, Lond. Edinb. Dubl. Phil. Mag. 34 (1849), 406-410.

[7] COCKLE, J.: On impossible equations, on impossible quantities, and on tesarines, Lond. Edinb. Dubl. Phil. Mag. 37 (1850), 281-283.

[8] PANDA, G.K.: Some fascinating properties of balancing numbers, In: Proc. Eleventh Internat. Conference on Fibonacci Numbers and Their Applications, Congr. Numer. Vol. 194 (2009), pp. 185-189.

[9] PANDA, G. K.: Sequence balancing and cobalancing numbers, Fibonacci Quart. 45 (2007), no. 3, 265-271.

[10] PANDA, G. K.-RAY, P. K.: Cobalancing numbers and cobalancers, Int. J. Math. Math. Sci. 8 (2005), 1189-1200.

[11] POGORUI, A. A.-RODRÍGUEZ-DAGNINO, R. M.-RODRÍGUEZ-SAID, R. D.: On the set of zeros of bihyperbolic polynomials, Complex Var. Elliptic Equ. 53 (2008), no. 7, 685-690.

[12] ROCHON, D.-SHAPIRO, M.: On algebraic properties of bicomplex and hyperbolic numbers, An. Univ. Oradea, Fasc. Mat. 11 (2004), 71-110.

[13] RAY, P. K.: Certain Matrices Associated with Balancing and Lucas-balancing Numbers, Matematika 28 (2012), no. 1, 15-22.

[14] RAY, P. K.- SAHU, J.: Generating functions for certain balancing and Lucas-balancing numbers, Palest. J. Math. 5 (2016), no. 2, 122-129.

Received April 7, 2020

\author{
Department of Discrete Mathematics \\ Faculty of Mathematics and Applied Physics \\ Rzeszow University of Technology \\ Powstańców Warszawy 12 \\ 35-959 Rzeszów \\ POLAND \\ E-mail: dorotab@prz.edu.pl \\ aszynal@prz.edu.pl \\ iwloch@prz.edu.pl
}

